\title{
The Diagnostic Value of Lung Ultrasonography in Children Community-Acquired Pneumonia
}

\author{
HENG LIAO*, XIAOJUAN ZHU AND YANDE NIU \\ Department of Ultrasound Medicine, Huzhou Central Hospital, Huzhou, Zhejiang 313000, China
}

Liao et al.: Lung Ultrasonography in Children Community-Acquired Pneumonia

\begin{abstract}
To explore the diagnostic value of lung ultrasonography in children community-acquired pneumonia is the objective of the study. A total of $\mathbf{1 2 6}$ children who were diagnosed with community-acquired pneumonia and underwent lung ultrasonography examination during the course of the disease were selected. All patients underwent lung ultrasonography and chest $X$-ray examination within $24 \mathrm{~h}$ of admission. The clinical characteristics of lung ultrasonography examination of community-acquired pneumonia were analyzed and the sensitivity, specificity, correct index and diagnostic coincidence rate of lung consolidation with lung ultrasonography and chest $\mathrm{X}$-ray were compared. The lung ultrasonography imaging features of severe community-acquired pneumonia in children are the disappearance of A-line, increase of B-line, waterfall sign, lung consolidation, bronchial inflation sign, pleural effusion, etc. 53 cases of patients chest computed tomography results were used as the gold standard. The sensitivity, correct index and diagnostic coincidence rate of lung ultrasonography in the diagnosis of lung consolidation were all higher than those of $\mathrm{X}$-ray diagnosis and the differences were statistically significant $(\mathbf{p}<\mathbf{0 . 0 5})$. Lung ultrasonography determined abnormal changes in the lungs of 124 children and chest $X$-ray determined abnormal changes in the lungs of 122 children. The chest $X$-rays of 2 children showed normal, while the lung ultrasonography showed a large number of B-line and lung consolidation in both lungs. A total of 17 cases of pleural effusion were determined by lung ultrasonography and 1 case of pleural effusion was determined by chest $X$-ray. The difference between the two groups was statistically significant $(p<0.05)$. Lung ultrasonography in children community-acquired pneumonia is superior to chest $\mathrm{X}$-ray in lung consolidation and pleural effusion and can be used for diagnosis of children community-acquired pneumonia.
\end{abstract}

Key words: Community-acquired pneumonia, lung ultrasonography, chest X-ray

Community-Acquired Pneumonia (CAP) is a common infectious disease in children, especially infants. It is the most common cause of hospitalization in children and the first cause of death in children under $5 \mathrm{y}$ of age $^{[1]}$. CAP is an acute infection that occurs in the lung parenchyma and/or interstitium. The clinical symptoms and signs include fever, wheezing, cough, dyspnea, inspiratory depression of chest wall, pulmonary moist rales, tubular breathing sounds, etc., accompanied by abnormal performance of Chest X-Ray (CXR). According to CAP management guidelines, CXR is the preferred imaging examination for the diagnosis of $\mathrm{CAP}^{[2,3]}$.

The lungs have been regarded as a forbidden area for ultrasound examination for a long time. Normal lung tissues contain good air. The ultrasound beam produces total reflection after reaching the pleura and cannot penetrate to the lung tissues. In recent years, with the advancement of ultrasound diagnosis technology, the ultrasound imaging of pleura and lung tissues is analyzed based on ultrasound artifacts under pathological conditions, making it possible to apply ultrasound to the diagnosis of CAP. In foreign countries, especially in developed countries, the application of ultrasound to diagnose various lung diseases has become a clinical research and application hotspot ${ }^{[4]}$, while the systemic ultrasound diagnosis of lung diseases in China is relatively $\operatorname{rare}^{[5]}$.

This study aimed to explore the clinical application value of Lung Ultrasonography (LUS) in the diagnosis of CAP. Through the ultrasound examination of 126 clinical cases diagnosed with CAP, the sonographic performance was analyzed and the examination experience and sonographic characteristics of CAP were obtained. 


\section{MATERIALS AND METHODS}

\section{Study subjects:}

A total of 126 children who were diagnosed with CAP in the pediatrics department of our hospital from September 2019 to March 2021, who underwent LUS examination during the course of the disease and met the inclusion and exclusion criteria were selected as the study subjects, aged from 2 mo to 14 y old, with an average age of $6.8 \pm 3.45 \mathrm{y}$, among which there were 66 males and 60 females.

Inclusion criteria: Children with clinical reasons (such as pneumonia and tumor) who had received chest Computed Tomography (CT) scan or needed to be hospitalized due to respiratory diagnosis results (such as pneumonia, shortness of breath, asthma, pleural effusion and para-pneumonia effusion).

Exclusion criteria: The time interval between LUS and CXR and/or lung CT examination was greater than $48 \mathrm{~h}$; children suffering from tumors, autoimmune diseases and severe immunodeficiency; children with congenital dysplasia of the lungs and thorax or with previous lung surgery; children suffering from congenital heart diseases, heart failure and severe liver and kidney diseases; existence of infection foci other than lung infection; newborn hospitalized patients.

\section{Methods:}

CXR: Under a supine position, a CXR by the bed was taken. The CXR was evaluated first by the radiologist on duty that day and then again evaluated by a radiology expert. The results of chest radiographs were classified according to the criteria of World Health Organization ${ }^{[6]}$.

LUS: Under sedation or in a quiet state, the bedside ultrasound examination was carried out in the supine and lateral position. The ultrasound instrument was Esaote MyLab Alpha, a portable ultrasound diagnostic instrument with a frequency of $3-12 \mathrm{MHz}$ linear probe. Operation method was as follows. Each side of the chest wall was divided into 12 areas, including the anterior upper, anterior lower, axillary upper, axillary lower, posterior upper and posterior lower, with the extension line of the parasternal line, anterior axillary line, posterior axillary line and double nipple connection. The ultrasound probe scanned each area one by one along the intercostal space horizontally or longitudinally. There were two professionally-trained sonographers to check and report the LUS.
Etiological test: Bacteria detection was done by $\mathrm{BD}$ Phoenix-100 automatic bacterial identification/drug susceptibility system was used to analyze specimens (sputum or alveolar lavage fluid) by automated analysis method. Virus and Mycoplasma pneumoniae detection by Throat swabs or alveolar lavage fluid was taken for fluorescent quantitative Polymerase Chain Reaction (PCR) detection.

\section{Statistical analysis:}

The data in this study was processed by Statistical Package for the Social Sciences (SPSS) 21.0 software. First, the Kolmogorov-Smirnov test method was used to test the normality of data in each group. The measurement data conforming to the normal distribution were expressed by the mean \pm standard deviation $(\mathrm{x} \pm \mathrm{s})$. The $t$ test was used for the comparison of measurement data between the two groups and analysis of variance followed by Tukey's method which was used for the comparison of measurement data among three groups and above. Non-normally distributed measurement data were expressed using median and quartile [M (q25, q75)]. The count data was described by rate or ratio. The comparison of count data was by chi-square test and the Bonferroni method was used to correct the test criteria when pairwise comparison was required, $\mathrm{p}<0.05$ indicated that the difference was statistically significant.

\section{RESULTS AND DISCUSSION}

Analysis of CAP LUS imaging results were shown here. Among the 126 CAP children, the LUS imaging showed that 2 cases were normal $(1.59 \%)$, disappearance of A-line was in 124 cases $(98.3 \%)$, increase of B-line was in 124 cases $(98.3 \%)$, waterfall sign was in 35 cases $(27.8 \%)$, lung consolidation was in 74 cases $(58.7 \%)$, bronchial inflation sign was in 30 cases $(22.5$ $\%)$, dynamic bronchial inflation sign was in 1 case $(0.8$ $\%$ ) and pleural effusion was in 17 cases $(13.4 \%)$, as shown in Table 1.

LUS and CXR in lung consolidation were compared. In this study, there were a total of 74 CAP cases whose LUS imaging indicated lung consolidation and 64 cases whose CXR examination showed lung consolidation. There was no statistically significant difference between the two groups ( $\mathrm{p}=0.217)$, as shown in Table 2 .

53 of the 126 patients underwent chest $\mathrm{CT}$ examination within $72 \mathrm{~h}$ after admission. When chest $\mathrm{CT}$ results were used as the gold standard, the sensitivity, correct index 
and diagnostic coincidence rate of LUS in diagnosing lung consolidation were higher than those of CXR and the differences were statistically significant $(\mathrm{z}=1.998$, $\mathrm{p}=0.0457 ; \mathrm{z}=1.998, \mathrm{p}=0.0457 ; \mathrm{z}=3.493, \mathrm{p}=0.0005$ ), as shown in Table 3. At the same time, 52 cases of chest CT results showed lung consolidation, among which 10 cases showed air bronchial shadow. LUS also showed 10 cases of lung consolidation with bronchial inflation signs, but CXR did not indicate.

LUS determined the location and distribution area of lung consolidation. In 74 cases determined by LUS examination, the lung consolidation was mainly concentrated on the back and the bilateral back was more common, accounting for $54 \%$. The rest were more common in the unilateral back and occasionally the front chest and side. Through LUS, there are 70 cases that described the area of lung consolidation in detail. The total area was $0.06-27.75 \mathrm{~cm}^{2}$, with a median of $2.05 \mathrm{~cm}^{2}$. The left was $0.06-14.70 \mathrm{~cm}^{2}$, with a median of $0.96 \mathrm{~cm}^{2}$. The right was $0.08-21.6 \mathrm{~cm}^{2}$, with a median of $1.8 \mathrm{~cm}^{2}$. There were 4 cases shown as several small pieces and the specific area was not described in detail, as shown in Table 4.

LUS and CXR in pleural effusion were compared. LUS determined 17 cases $(13.5 \%)$ of pleural effusion, while CXR determined only 1 case $(0.08 \%)$. The difference between the two groups was statistically significant $(\mathrm{p}=0.011$ ), as shown in Table 5. Among 53 patients determined by chest CT, 2 cases of pleural effusion were determined by chest CT, 7 cases were determined by LUS and 0 cases were determined by CXR. Through chest $\mathrm{CT}$, there were 8 cases that showed local pleural adhesions, but CXR and LUS did not indicate. The results of LUS revealed the location of pleural effusion in 17 cases, 10 cases on both sides, 5 cases on the left and 2 cases on the right. The pleural effusion minimum was $1 \mathrm{~mm}$.

The etiological characteristics between the consolidation group and the non-consolidation group were compared. The LUS results in the consolidation group and the non-consolidation group were compared in terms of simple bacterial infection, simple viral infection, mixed infection and drug-resistant bacteria and the differences

\section{TABLE 1: LUS RESULTS OF CHILDREN CAP}

\begin{tabular}{lc}
\hline LUS results & $\mathbf{N}(\%)$ \\
\hline Normal & $2(1.59)$ \\
Disappearance of A-line & $124(98.4)$ \\
Increase of B-line & $124(98.4)$ \\
Waterfall sign & $35(27.8)$ \\
Lung consolidation & $74(58.7)$ \\
Bronchial inflation sign & $30(23.8)$ \\
Dynamic bronchial inflation sign & $1(0.8)$ \\
Pleural effusion & $17(13.4)$ \\
\hline
\end{tabular}

TABLE 2: LUS AND CXR IN LUNG CONSOLIDATION

\begin{tabular}{lccc}
\hline & \multicolumn{2}{c}{ LUS } \\
\hline CXR & Positive & Negative & Total \\
Positive & 41 & 23 & 64 \\
Negative & 33 & 29 & 62 \\
Total & 74 & 52 & 126 \\
\hline
\end{tabular}

TABLE 3: THE DIAGNOSTIC EFFECTS OF THE TWO EXAMINATION METHODS USING CHEST CT AS THE GOLD STANDARD

\begin{tabular}{lcccc}
\hline Examination methods & Sensitivity & Specificity & Correct index & $\begin{array}{c}\text { Diagnostic coincidence } \\
\text { rate }\end{array}$ \\
\hline CXR & $40.4 \%(21 / 52)$ & $100 \%(1 / 1)$ & 0.404 & $41.5 \%(22 / 52)$ \\
LUS & $59.6 \%(31 / 52)$ & $100 \%(1 / 1)$ & 0.596 & $60.4 \%(32 / 52)$ \\
& 1.998 & - & 1.998 & 3.493 \\
$\mathrm{p}$ & 0.0457 & & 0.0457 & 0.0005 \\
\hline
\end{tabular}


TABLE 4: LUS SHOWED THE DISTRIBUTION AREA OF LUNG CONSOLIDATION

\begin{tabular}{lccc}
\hline & Total (\%) & Left $(\mathbf{N})$ & Right $(\mathbf{N})$ \\
\hline$<1 \mathrm{~cm}^{2}$ & $26(37.1)$ & 29 & 18 \\
$1-3 \mathrm{~cm}^{2}$ & $14(25)$ & 17 & 20 \\
$3.01-6 \mathrm{~cm}^{2}$ & $15(21)$ & 7 & 12 \\
$6.01-10 \mathrm{~cm}^{2}$ & $7(9)$ & 3 & 3 \\
$10-20 \mathrm{~cm}^{2}$ & $6(7.2)$ & 2 & 2 \\
$>20 \mathrm{~cm}^{2}$ & $2(1.8)$ & 0 & 1 \\
\hline
\end{tabular}

TABLE 5: LUS AND CXR IN PLEURAL EFFUSION

\begin{tabular}{lccc}
\hline & \multicolumn{2}{c}{ LUS } \\
\hline CXR & Positive & Negative & Total \\
Positive & 1 & 0 & 1 \\
Negative & 16 & 109 & 125 \\
Total & 17 & 109 & 126 \\
\hline
\end{tabular}

TABLE 6: THE ETIOLOGICAL CHARACTERISTICS IN THE CONSOLIDATION GROUP AND THE NONCONSOLIDATION GROUP

\begin{tabular}{lcccc}
\hline & $\begin{array}{c}\text { Simple bacterial } \\
\text { infection }(\mathrm{N})\end{array}$ & $\begin{array}{c}\text { Simple viral infection } \\
(\mathrm{N})\end{array}$ & $\begin{array}{c}\text { Mixed infection }(\mathrm{N}) \\
\text { Consolidation group }\end{array}$ & $\begin{array}{c}\text { Drug-resistant bacteria } \\
(\mathrm{N})\end{array}$ \\
$\begin{array}{l}\mathrm{n}=74) \\
\text { Non-consolidation }\end{array}$ & 17 & 11 & 7 & 7 \\
group & 13 & 10 & 5 & 2 \\
$\mathrm{p}$ & 0.699 & 0.455 & 0.920 & 0.250 \\
\hline
\end{tabular}

were not statistically significant $(\mathrm{p}=0.699, \mathrm{p}=0.455$, $\mathrm{p}=0.920, \mathrm{p}=0.250$ ), as shown in Table 6 .

Children CAP refer to infectious pneumonia acquired by healthy children outside the hospital, which is one of the important causes of death in children under $5 \mathrm{y}$ of age ${ }^{[7]}$. At present, the diagnosis of children CAP is mainly based on medical history, clinical manifestations, laboratory examinations, imaging examinations, etc. Imaging examinations are still mainly focused on CXR and chest $\mathrm{CT}$. Because the completion of chest $\mathrm{CT}$ needs to move the child, it is not conducive to the recovery of the child to some extent, unless special diseases such as bronchial foreign bodies, congenital pulmonary dysplasia, etc., need to be identified ${ }^{[8]}$. Because anteroposterior chest radiographs can be performed at the bedside and the latest guidelines point out that for CAP children that need to be judged for pneumonia complications or exacerbations, CXR radiographs should be performed in time ${ }^{[9]}$, so chest radiographs are often applied in children intensive care unit. However, chest radiographs and chest $\mathrm{CT}$ examinations are both radioactive. A study has shown that due to long life expectancy and high tissue radiosensitivity, the risk of fatal cancer in children is not negligible ${ }^{[10]}$; there are also data showing that the incidence and mortality of cancer in children due to radiation are 3 to 5 times higher compared with those in adults ${ }^{[11,12]}$ and the risk of radiation is cumulative: each examination will increase the dose and promote the $\operatorname{risk}^{[13]}$. As a new imaging technique, LUS has been gradually applied to lung diseases in adults, children and newborns in recent years. Normal people's alveoli contain air and the acoustic impedance of the air is very large, blocking the penetration of ultrasound. Ultrasound is reflected multiple times between the probe and the pleura, forming a series of characteristic "artifacts", making it impossible to see the real structure in the lungs. However, when there is disease in the lungs and the lung tissues are filled with some exudates, blood, etc., the lung air content will change. At this time, ultrasound can penetrate into the lungs through the pleura, forming some disease-specific signs. Related reports have confirmed that LUS can be used as a reliable tool for the diagnosis of adult pneumonia ${ }^{[10,11]}$. Parlamento et al. have shown that LUS is more sensitive than chest radiographs in emergency department patients diagnosed with pneumonia ${ }^{[14]}$. Copetti et al. also have showed that LUS is more sensitive to the diagnosis 
of children pneumonia than chest radiographs ${ }^{[15]}$. The International Joint Committee on Lung Ultrasound even has pointed out, "lung ultrasound is as accurate as a chest radiograph in the diagnosis of pneumonia in children" ${ }^{[2]}$. However, there are few studies on children $\mathrm{CAP}$ at home and abroad.

In the etiological test results of this study, bacteria accounted for $58.3 \%$, viruses accounted for $38 \%$ and other pathogens accounted for $14.5 \%$. In etiology, respiratory syncytial virus was the most common. The top 3 bacteria determined were Streptococcus pneumoniae, Staphylococcus aureus and Haemophilus influenzae, which is consistent with domestic and foreign research ${ }^{[16]}$. However, this study demonstrated the existing research on the detection rate of Staphylococcus aureus and Haemophilus influenzae $e^{[13]}$, indicating that the above-mentioned bacteria are more likely to be infected with children CAP, which may provide certain help in the selection of antibiotics in the future. Respiratory syncytial virus infection is the main viral infection, which is consistent with previous reports. Combined with the age characteristics of this study, it suggested that respiratory syncytial virus infection in children CAP is still predominant in infants. At the same time, 3 cases of Mycoplasma infection were found and the detection rate was $3.4 \%$, which was significantly lower than that in domestic and foreign related studies ${ }^{[17,18]}$. This may be related to the selection criteria of Mycoplasma pneumoniae infection in this study, while previous studies have mostly selected Mycoplasma antibody positive as the selection criteria. At the same time, many factors such as age, region, climate, etc., cannot be ruled out.

This study focused on the early diagnosis of children CAP and LUS was performed. In addition to pneumonia and LUS findings that may alter lung dynamics, patients with other diseases were excluded from the study. This is similar to the research method of Guerra and Heffeman abroad ${ }^{[19]}$. The main pathological changes of children pneumonia are congestion and edema of lung tissues. The alveoli are gradually filled with infiltrated inflammatory cells, exudates, etc., forming a patchy inflammatory lesion. When the lesion is further fused into a piece, multiple pulmonary lobules or more may be involved. When the small bronchi and bronchiole are inflamed, it can lead to partial or complete blockage of the lumen, causing emphysema or atelectasis. A number of previous studies have revealed that when lung inflammation occurs, LUS manifests as the increase of B-line, waterfall sign, lung consolidation, bronchial inflation sign and pleural effusion ${ }^{[20,21]}$. This study demonstrated that LUS changes in children CAP were consistent with previous studies and there were no other ultrasound images of the lungs due to severe illness. In this study, the LUS imaging of 124 CAP children showed abnormalities. Among them, 2 LUS imaging showed increase of B-line and lung consolidation, while CXR showed normal and 1 LUS imaging showed a small amount of B-line. The chest radiographs showed that the texture of both lungs was thickened, which is basically consistent with the results of Yilmaz et al. ${ }^{[16]}$. When the alveoli are congested with edema, the water content in the lungs increases and increase of B-line can be seen on the LUS. This study revealed that in CAP children, the number of cases with increase of B-line upregulated significantly and 35 cases had waterfall sign. A study has shown that there is important clinical information about the distance between the $\mathrm{B}$ lines. The distance between the B lines is about $3 \mathrm{~mm}$ or fewer (called the B3 line), which is caused by ground glass lesions under the pleura, indicating alveolar edema; the distance between the B lines is about $7 \mathrm{~mm}$ (called the B7 line), which is caused by the thickened subpleural lobule interval, suggesting interstitial edema ${ }^{[22]}$. There is also a report showing that the number of B-lines determines the degree of pulmonary ventilation deficiency, the larger the number of B-lines, the more severe the degree of pulmonary ventilation deficiency. This also indirectly suggests that almost all CAP children have poor pulmonary ventilation.

When the air content of the alveoli is further reduced, the lung tissues are solidified and the sound image can be regarded as a solid tissue similar to the echo of the liver and spleen, which indicates lung consolidation. In this study, CXR and LUS were basically the same in the detection rate of lung consolidation. There was no statistically significant difference between the two groups. However, when chest CT is used as the gold standard, the sensitivity, correct index and diagnostic coincidence rate of lung consolidation in LUS detection are higher than CXR diagnosis, which also suggests that LUS is superior to CXR in diagnosing children CAP. Moreover, because CAP children are often seriously ill, it is possible to take multiple CXRs during the hospitalization and thus receive multiple radiations, which may cause the risk of cancer in the future. The LUS has the characteristics of non-radiation and is more suitable for children intensive care unit. A report indicates that LUS also has its shortcomings for that the detection rate of consolidation that cannot reach the 
pleura is low and smaller consolidations $(<1$ intercostal space) may also appear ${ }^{[23]}$. The chest radiographs may be missed due to the blocking of some lung tissues by the heart, diaphragm, ribs, etc. LUS examination results are negative, but the chest radiograph results are positive. The possible reasons are as follows: The lung consolidation does not reach the surface of the pleura (usually located around the hilar and paracardia) [24,25]; the lung consolidation is located in areas that are difficult to contact with LUS, such as the scapula, supraclavicular area or axillary area ${ }^{[26]}$. Lissama et al. has found that LUS has a high specificity for lung consolidation over $1 \mathrm{~cm}^{[27]}$. This study demonstrated that the lung consolidation area determined by LUS was concentrated within $1 \mathrm{~cm}^{2}$, with the smallest area being $0.06 \mathrm{~cm}^{2}$, suggesting that lung consolidation $<0.06 \mathrm{~cm}^{2}$ may be missed. However, bedside chest radiographs are not particularly accurate in describing the location and size of lung consolidation. In this study, the chest radiograph report mostly used words such as "by the lungs", "beside the heart shadow", etc., to describe, while the consolidation area of the lungs was only described as patchy and the specific size was not able to be described. The sonographer can directly communicate with clinicians during bedside LUS examinations for CAP children and then directly understand the location and size of the children's lung consolidation and can observe and evaluate pulmonary ventilation in real time. Thus, compared with the anteroposterior chest radiograph, LUS may be more instructive for follow-up treatment and dynamic observation. In this study, it was found that the lung consolidation was mainly concentrated on the back and the bilateral back was more common. This may be because the pathological type of pneumonia in children is mostly lobular pneumonia, which is more common in bilateral lower lobe and dorsal side. At the same time, it may also be due to the fact that most children in intensive care are in the supine position and the LUS is not disturbed by the heart, diaphragm and other tissues during the back examination and the lung tissues are scanned more accurately, so as to obtain clearer ultrasound images.

LUS is more advantageous in detecting pleural effusion. The results of this study showed that the detection rate of pleural effusion of CXR was significantly lower than that of LUS and the difference was statistically significant, suggesting that LUS was significantly better than CXR in the detection of pleural effusion. CXR only determined 1 case of pleural effusion, which was located on the right, but the results of LUS examination of the same child indicated that there were pleural effusions on both sides. In this study, LUS was able to detect at least $1 \mathrm{~mm}$ of pleural effusion, which was more common on both sides. In addition, it is reported that compared with CT scans, LUS is more sensitive to the diagnosis of micropleural and pleural effusion ${ }^{[23]}$. In the 53 cases of chest CT examination results in this study, LUS showed significantly more cases of pleural effusions than chest CT and CXR did, which is also consistent with previous research results ${ }^{[23]}$. In the past, the examination posture for pleural effusion was mostly upright or sitting, because gravity can deposit pleural effusion on the bottom of the lungs. The upright position is more helpful for the detection of pleural effusion. However, due to the particularity of the pediatric intensive care unit, the patients enrolled this time were examined in the supine position, which also indicates that LUS still has its high resolution for pleural effusion in the supine position. Moreover, in the diagnostic criteria for the severity of children CAP in the United States, pleural effusion is clearly pointed out as a scoring standard. It can be inferred that LUS is superior to chest radiographs in the diagnosis of children CAP.

In conclusion, the characteristics of LUS imaging in children CAP are the disappearance of A-line, increase of B-line, waterfall sign, lung consolidation, bronchial inflation sign, pleural effusion, etc. In terms of lung consolidation and pleural effusion, LUS is superior to CXR and has the advantages of simplicity, no radiation, being able to be performed at the bedside, etc. It can be used to diagnose children CAP and is more suitable for intensive care units, reducing radiation exposure, but has no significant characteristics in etiology.

\section{Acknowledgements:}

This work was supported by the Public Welfare Application Research Project of Huzhou Science and Technology Bureau (No. 2019GY32).

\section{Conflict of interests:}

The authors declared no conflict of interest.

\section{REFERENCES}

1. Caiulo VA, Gargani L, Caiulo S, Fisicaro A, Moramarco F, Latini G, et al. Lung ultrasound in bronchiolitis: Comparison with chest X-ray. Eur J Pediatr 2011;170(11):1427-33.

2. Volpicelli G, Elbarbary M, Blaivas M, Lichtenstein DA, Mathis G, Kirkpatrick AW, et al. International evidence-based recommendations for point-of-care lung ultrasound. Intensive Care Med 2012;38(4):577-91. 
3. Lichtenstein DA. Lung ultrasound in the critically ill. Ann Intensive Care 2014;4(1):1-2.

4. Tsung JW, Kessler DO, Shah VP. Prospective application of clinician-performed lung ultrasonography during the 2009 H1N1 influenza A pandemic: Distinguishing viral from bacterial pneumonia. Crit Ultrasound J 2012;4(1):1-10.

5. Corradi F, Brusasco C, Pelosi P. Chest ultrasound in acute respiratory distress syndrome. Curr Opin Crit Care 2014;20(1):98-103.

6. Hu QJ, Shen YC, Jia LQ, Guo SJ, Long HY, Pang CS, et al. Diagnostic performance of lung ultrasound in the diagnosis of pneumonia: A bivariate meta-analysis. Int J Clin Exp Med 2014;7(1):115-21.

7. Liu J, Liu F, Liu Y, Wang HW, Feng ZC. Lung ultrasonography for the diagnosis of severe neonatal pneumonia. Chest 2014;146(2):383-8.

8. Cardenas-Garcia J, Mayo PH. Bedside ultrasonography for the intensivist. Crit Care Clin 2015;31(1):43-66.

9. Pereda MA, Chavez MA, Hooper-Miele CC, Gilman RH, Steinhoff MC, Ellington LE, et al. Lung ultrasound for the diagnosis of pneumonia in children: A meta-analysis. Pediatrics 2015;135(4):714-22.

10. Basile V, di Mauro A, Scalini E, Comes P, Lofù I, Mostert $\mathrm{M}$, et al. Lung ultrasound: A useful tool in diagnosis and management of bronchiolitis. BMC Pediatr 2015;15(1):1-8.

11. Riviello ED, Kiviri W, Twagirumugabe T, Mueller A, BannerGoodspeed VM, Officer L, et al. Hospital incidence and outcomes of the acute respiratory distress syndrome using the Kigali modification of the Berlin definition. Am J Respir Crit Care Med 2016;193(1):52-9.

12. Osvald EC, Clarke JR. NICE clinical guideline: Bronchiolitis in children. Arch Dis Child Educ Pract Ed 2016;101(1):46-8.

13. Varshney T, Mok E, Shapiro AJ, Li P, Dubrovsky AS. Point-ofcare lung ultrasound in young children with respiratory tract infections and wheeze. Emerg Med J 2016 ;33(9):603-10.

14. Parlamento S, Copetti R, di Bartolomeo S. Evaluation of lung ultrasound for the diagnosis of pneumonia in the ED. Am J Emerg Med 2009;27(4):379-84.

15. Copetti R, Cattarossi L. Ultrasound diagnosis of pneumonia in children. Radiol Med 2008;113(2):190-8.

16. Yilmaz HL, Özkaya AK, Gökay SS, Kendir ÖT, Şenol H. Point-of-care lung ultrasound in children with community acquired pneumonia. Am J Emerg Med 2017;35(7):964-9.

17. Wang Y, Gargani L, Barskova T, Furst DE, Cerinic MM. Usefulness of lung ultrasound B-lines in connective tissue disease-associated interstitial lung disease: A literature review. Arthritis Res Ther 2017;19(1):1-9.
18. Omran A, Eesai S, Ibrahim M, El-Sharkawy S. Lung ultrasound in diagnosis and follow up of community acquired pneumonia in infants younger than 1-year old. Clin Respir J 2018;12(7):2204-11.

19. See KC, Ong V, Tan YL, Sahagun J, Taculod J. Chest radiography versus lung ultrasound for identification of acute respiratory distress syndrome: A retrospective observational study. Crit Care 2018;22(1):1-9.

20. Jaszczolt S, Polewczyk T, Dolega-Kozierowska M, Woźniak M, Doniec Z. Comparison of lung ultrasound and chest $\mathrm{X}$-ray findings in children with bronchiolitis. J Ultrason 2018;18(74):193-7.

21. Biagi C, Pierantoni L, Baldazzi M, Greco L, Dormi A, Dondi A, et al. Lung ultrasound for the diagnosis of pneumonia in children with acute bronchiolitis. BMC Pulm Med 2018;18(1):1-10.

22. Supino MC, Buonsenso D, Scateni S, Scialanga B, Mesturino MA, Bock C, et al. Point-of-care lung ultrasound in infants with bronchiolitis in the pediatric emergency department: A prospective study. Eur J Pediatr 2019;178(5):623-32.

23. Trinavarat P, Riccabona M. Potential of ultrasound in the pediatric chest. Eur J Radiol 2014;83(9):1507-18.

24. Claes AS, Clapuyt P, Menten R, Michoux N, Dumitriu D. Performance of chest ultrasound in pediatric pneumonia. Eur J Radiol 2017;88:82-7.

25. Urbankowska E, Krenke K, Drobczyński Ł, Korczyński P, Urbankowski T, Krawiec M, et al. Lung ultrasound in the diagnosis and monitoring of community acquired pneumonia in children. Respir Med 2015;109(9):1207-12.

26. Ho MC, Ker CR, Hsu JH, Wu JR, Dai ZK, Chen IC. Usefulness of lung ultrasound in the diagnosis of community-acquired pneumonia in children. Pediatr Neonatol 2015;56(1):40-5.

27. Lissaman C, Kanjanauptom P, Ong C, Tessaro M, Long E, O'Brien A. Prospective observational study of point-ofcare ultrasound for diagnosing pneumonia. Arch Dis Child 2019;104(1):12-8.

This is an open access article distributed under the terms of the Creative Commons Attribution-NonCommercial-ShareAlike 3.0 License, which allows others to remix, tweak, and build upon the work non-commercially, as long as the author is credited and the new creations are licensed under the identical terms

This article was originally published in a special issue, "Novel Therapeutic Approaches in Biomedicine and Pharmaceutical Sciences" Indian J Pharm Sci 2021:83(6) Spl Issue "255-261" 